THE KURUME MEDICAL JOURNAL Vol. 16, No. 1, 1969

\title{
FLUORESCEIN FUNDUS PHOTOGRAPHIC STUDIES ON THE CENTRAL SEROUS RETINOPATHY
}

\author{
Part II Fluorescein fundus photographic findings \\ in the full course of this disease
}

\author{
HISAHARU YOSHIOKA AND TAKASHI SUGITA \\ Department of Ophthalmology, Kurume University School of Medicine, \\ Kurume, Japan
}

(Received for publication February 24, 1969)

There are several excellent reports ${ }^{11-5)}$ on fluorescein fundus photographic findings in the central serous retinopathy. However, the full course from onset to cure of this disease are not yet examined in the same individual. Fujisawa $(1966)^{2)}$ has observed the course of seven cases with central serous retinopathy and divided it into three stages; early stage, middle stage, and last stage.

However, he has not mentioned on the detachment of the retinal pigment epithelium. Otherwise, Gass (1967) ${ }^{3}$ ) has pointed out the detachment of the retinal pigment epithelium in these disease but unfortunately the serial fluorescein fundus angiographic findings in the course of this disease have not been cleared.

The purpose of present paper is to report the repeatedly examined fluorescein fundus angiographic feature in the full course of this disease in the same individual.

\section{CASE REPORTS}

Case 1. 40-year-old man

He noted sudden onset of a slanting vision, metamorphopsia, micropsia, and central scotoma in the left eye ten days before. Visual acuity in the left eye on the initial examination was $0.5 \mathrm{with}+1.0 \mathrm{D} \mathrm{sph}$. Funduscopic examination revealed loss of foveal reflex and there was a circumscribed area of serous detachment of the macula (Fig. 1). Slit lamp examination revealed a localized serous detachment of the macular retina. There was a number of a yellowish-white subretinal precipitates. There was also evidence of pigment epithelium detachment temporal to the foveal region. One month after onset of ocular signs, his vision in left eye is estimated 0.7 uncorrected. At this time, review of his funduscopy revealed still loss of foveal reflex and a circumscribed macular edema. There were many faint yellowish-gray punctate deposits in one disc diameter temporal to the fovea. And also there was an area of pigmentary disturbance inferior to the fovea.

Fluorescein fundus findings. - 13th day after onset; the mound of the round 


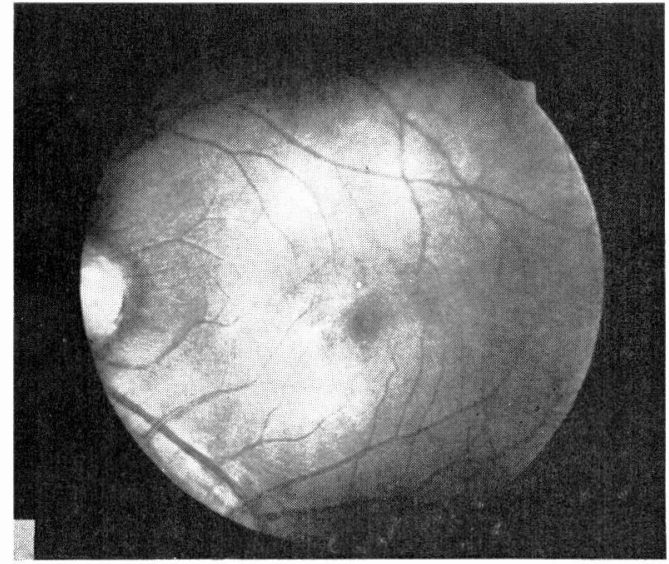

Fig. 1. Case 1. left eye. A circumscribed elevation of macular retina. There was a pigment epithelium detachment temporal superior to the foveal region.

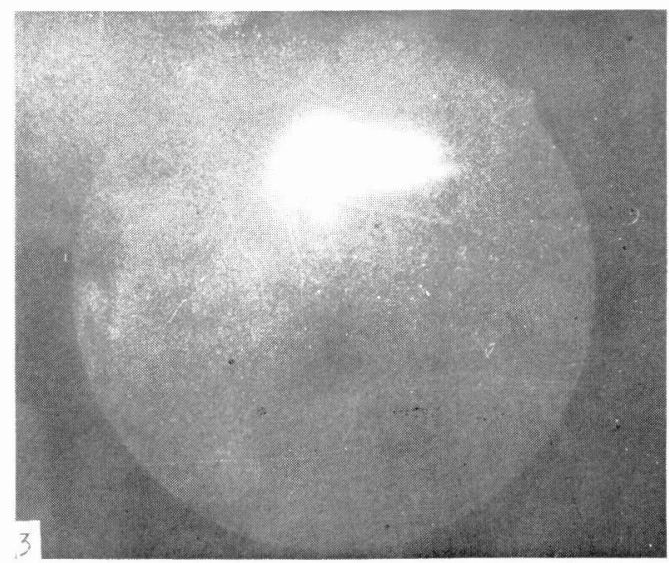

Fig. 3. Jetting spot slightly enlarge right side in the superior margin of detached retina in thirty minutes after injection.

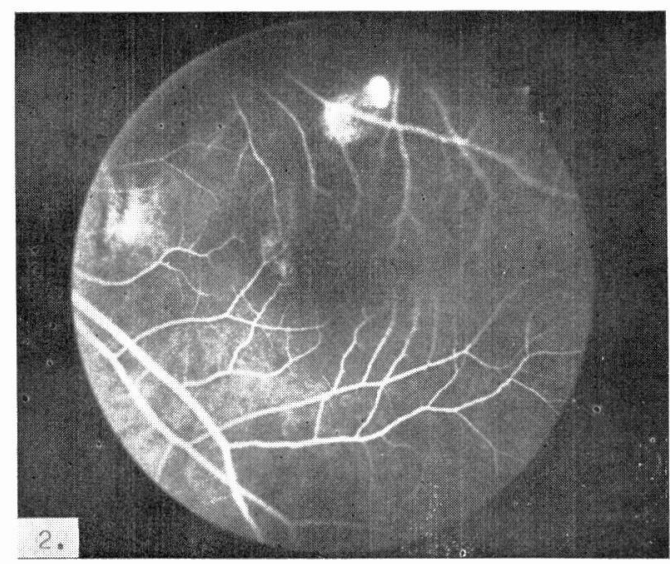

Fig. 2. Case 1. 13th day aiter onset; the mound of the nearly round abnormal fluorescence corresponding of area in pigment epithelium detachment.

In arteriovenous phase, dense spot of fluorescence appear in this and in time the dye jet superiorly.

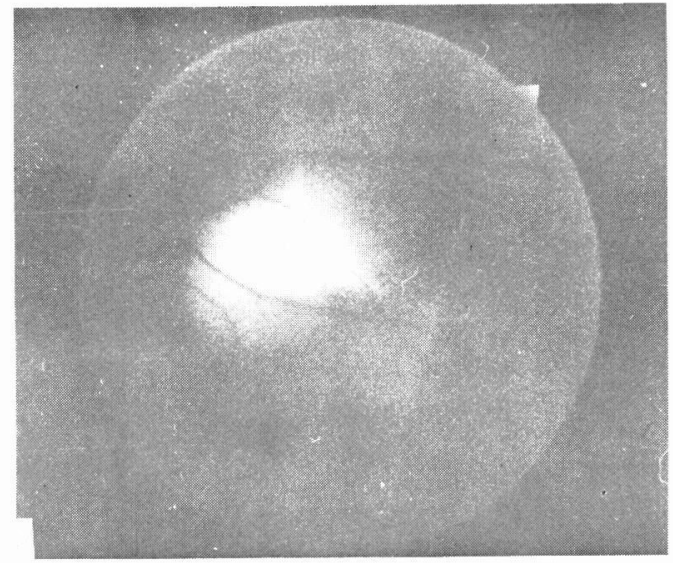

Fig. 4. One hour later, the dye become to nearly fill the entire subretinal space.

abnormal fluorescence which has middle size and mottled appearance appear in the area of one disc diameter from the fovea and temporal inferior to the fovea in arterial phase. This mound is seem to be an area of pigment epithelium detachment. The area of the nasal inferior half to this mound show denser fluorescence than the area of the temporal superior half (Fig. 2). In arteriovenous phase, dense spot of fluorescence appear in the faint part of this mound and in 


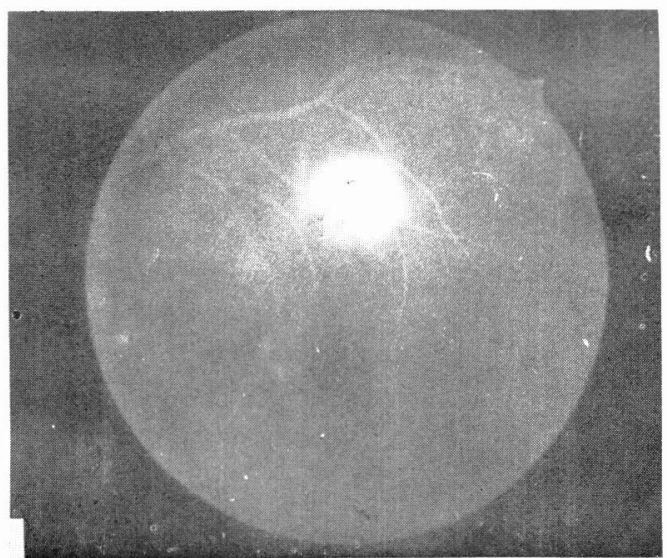

Fig. 5. Case 1. 40th day after onset; in venous phase, spot previously jeted show the dense fluorescence and this spot of fluorscence enlarge concentrically in time.

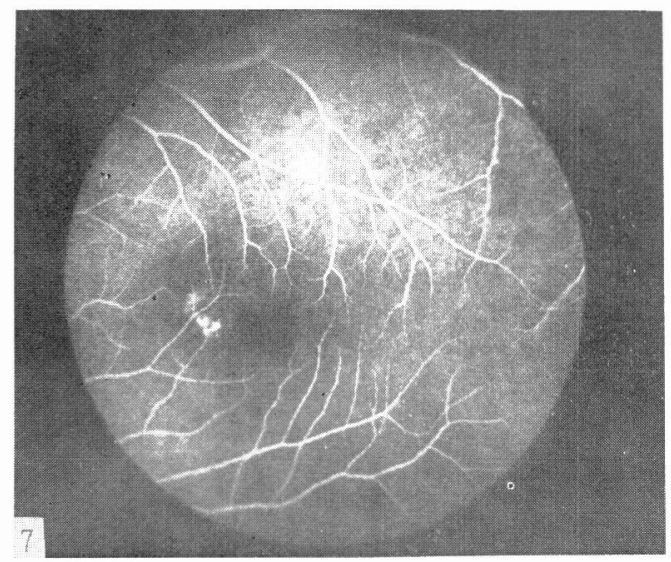

Fig. 7. Case 1. 4 months after onset; the spot of fluorescence previously round enlarged slightly increase in density and do not alter in shape and size.

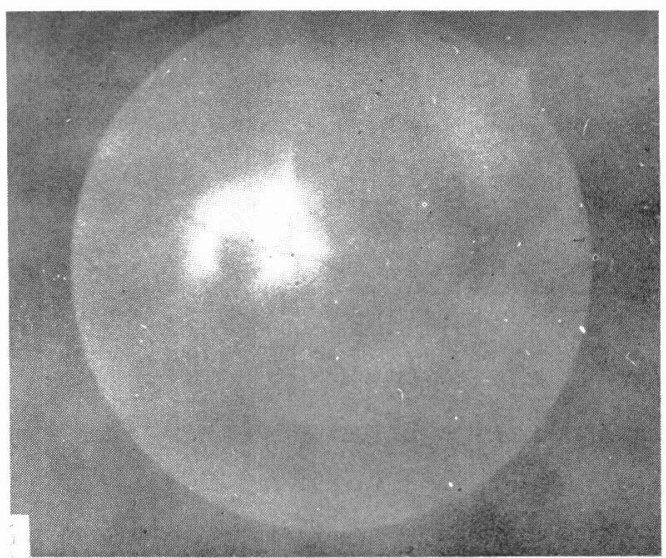

Fig. 6. Sixty minutes later, the dye become to fill the entire detached retina.

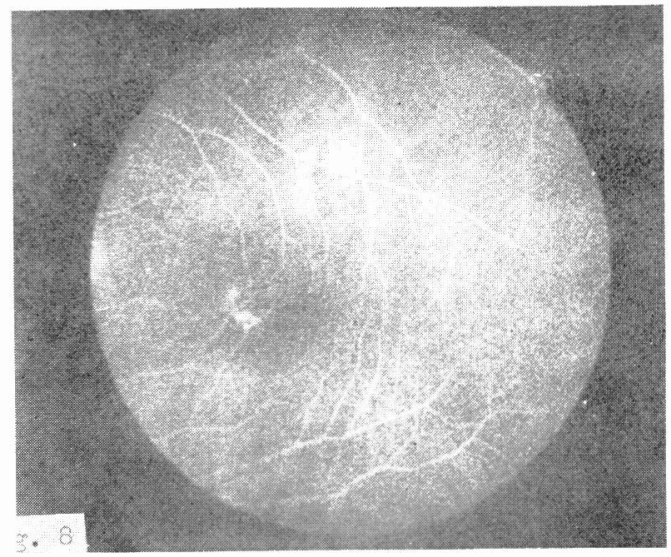

Fig. 8. This spot of fluorescence remain constantly after the backgraund fluorescence has disappeared.

time, the dye jet superiorly from the spot to subretinal space. Thirty minutes later, the dye become to fill the entire subretinal space corresponding to the detached retina (jet type) (Fig. 3, 4). 40th day after onset; the mound of fluorescence with mottled appearance appear in the same area of the first examination in the arterial phase. Differing from the finding of the first examination, the mound uniformly fluorescence. In arteriovenous phase, spot previously jeted show the dense fluorescence but do not jet superiorly. This spot of 


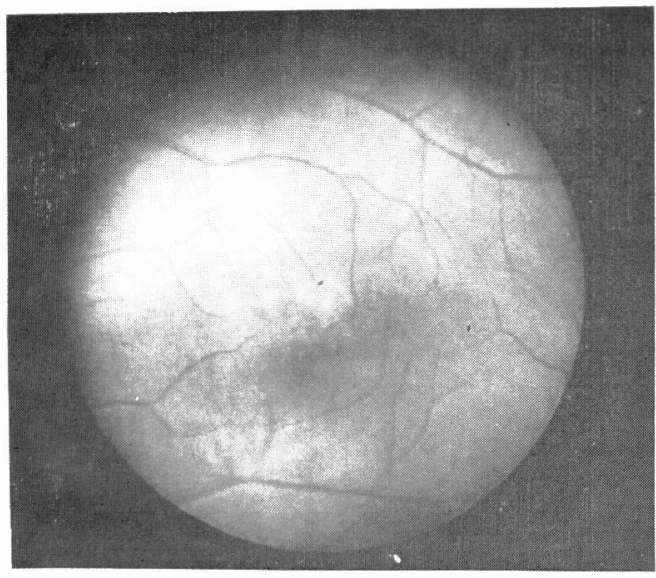

Fig. 9. Case 2. left eye. A circumscribed round edema in the macular region.

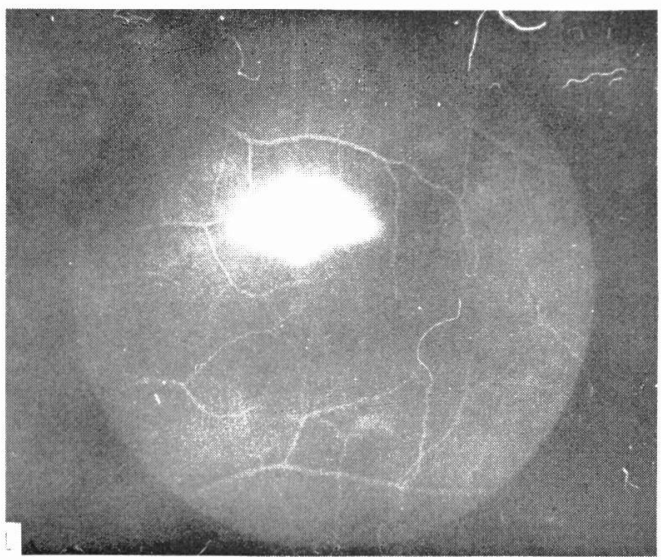

Fig. 11. Jeting spots slightly enlarge both right and left side in the superior margin of detached retina in five minutes later.

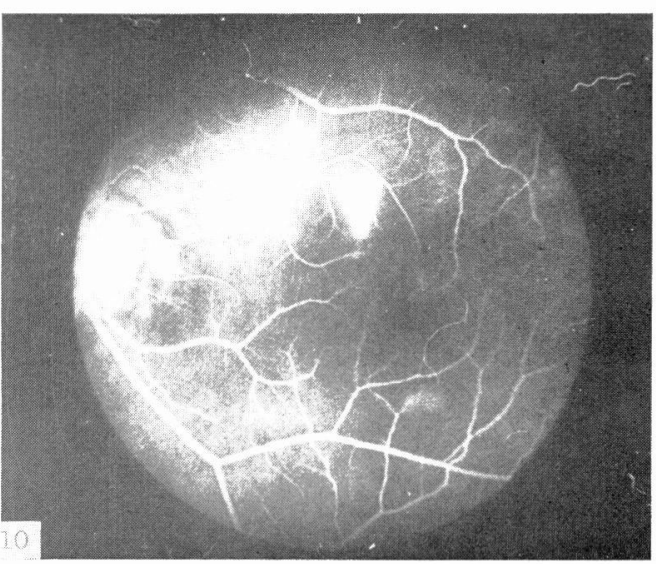

Fig. 10. Case 2. 17th day after onset; the spot appears with the focal detachment of pigment epithelium. The dye jets from this spot superiorly in time.

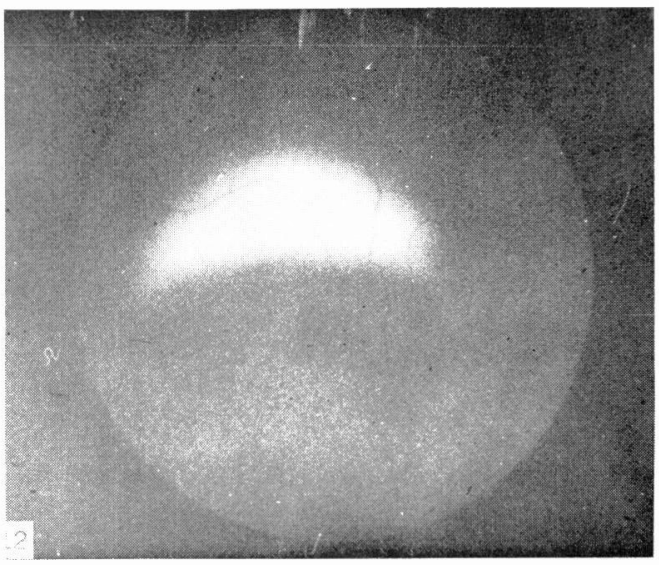

Fig. 12. The dye fill the superior half of detached retina in thirty minutes later.

fluorescence enlarge concentrically in time and the dye become to fill the entire detached retina sixty minutes later (enlarged type) (Fig. 5, 6). 4 months after onset; the mound of fluorescence with mottled appearance appear in the same area in arterial phase. The spot of fluorescence previously round enlarged slightly increase in density and do not alter in shape and size. This spot of fluorescence remain constantly after the background fluorescence has disappeared (residual type) (Fig. 7, 8). 
Case 2. 37-year-old man

He developed sudden onset of blurred vision in the left eye. At the same time, he noted metamorphopsia and central scotoma. His visual acuity in the left eye was 0.4 uncorrected. Ophthalmoscopy revealed a circumscribed round edema in the macular region. Foveal reflex is absent (Fig. 9). Focal retinal pigment epithelium detachment could not be visualized on slit lamp examination. Forty days later, the serous retinal detachment almost disappeared in the left fundus. The foveal reflex was unclear. There were several small yellowish punctate deposits in the parafoveal area.

Fluorescein fundus findings. - 17th day after onset; the focal type of abnormal fluorescence appear just nasal superior to the fovea in arterial phase. Three seconds later, the spot within the focal fluorescence increase in density and the dye jets from this spot superiorly in time (Fig. 10). At the same time, jeting spot slightly enlarge both right and left side in the superior margin of detached retina and gradually descend inferiorly (Fig. 11). Thirty minutes later, the dye fill the superior half of the detached retina but become to fill the entire detached retina in sixty minutes later (Fig. 12). 25th day after onset; focal type of abnormal fluorescence appear in arterial phase same as the first examination.

The spot within the focal fluorescence increase in density and begin to jet temporal inferiorly from this spot in this time. In time, an previously jeting area gradually enlarge to describe a parabola. The fluorescence of its tip is smaller than those of jeting area and fluorescence of its tip increase in size (Fig. 13, 14). Sixty minutes later, the dye become to fill the entire detached retina. 59th day after onset; fluorescein leakage do not show jet type but gradually enlarge concentrically in the spot in this period. In thirty one seconds after injection,

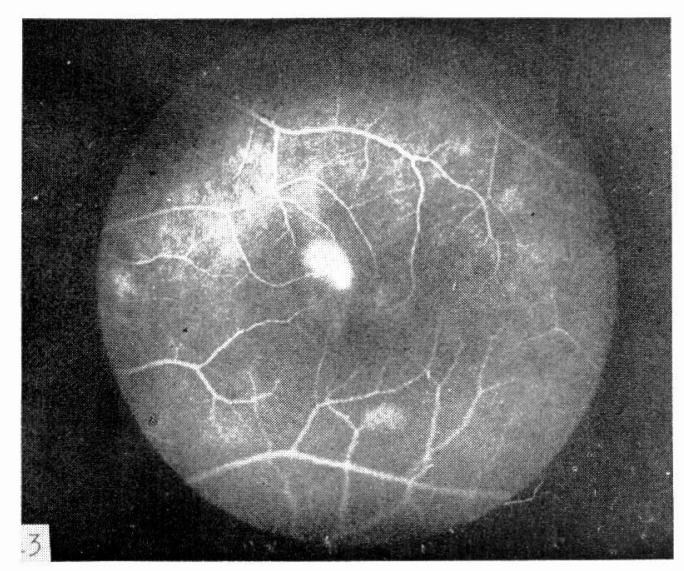

Fig. 13. Case 2. 25th day after onset, the dye begin to jet temporal inferior in arteriovenous phase.

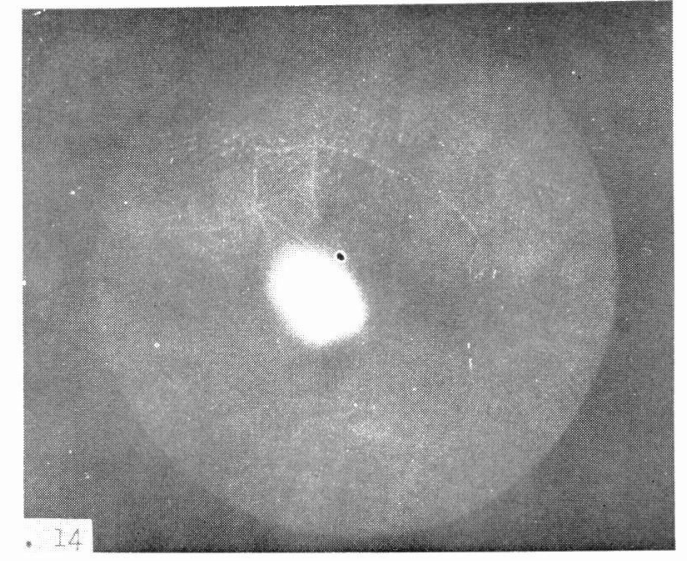

Fig. 14. 5 minutes after injection, jeting area gradually enlarge to deseribe a parabola and the dye become to fill the entire detached retina. 


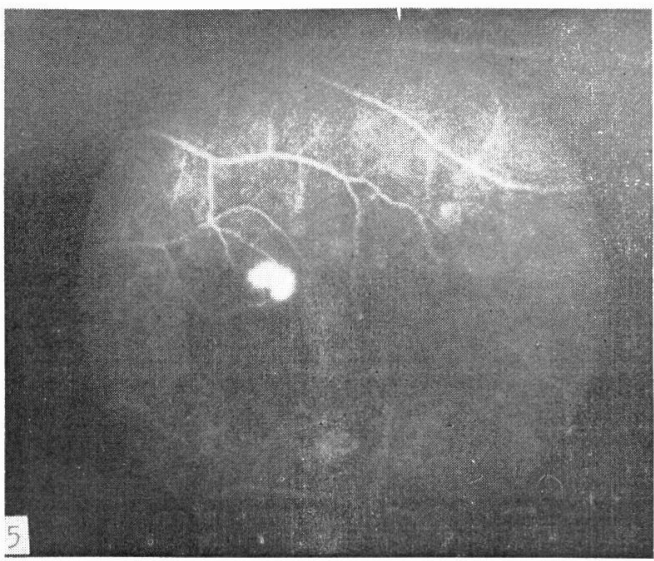

Fig. 15. Case 2. 59th day after onset, in thirty one seconds after injection, dye in this spot begin to jet minimally temporal inferiorly.

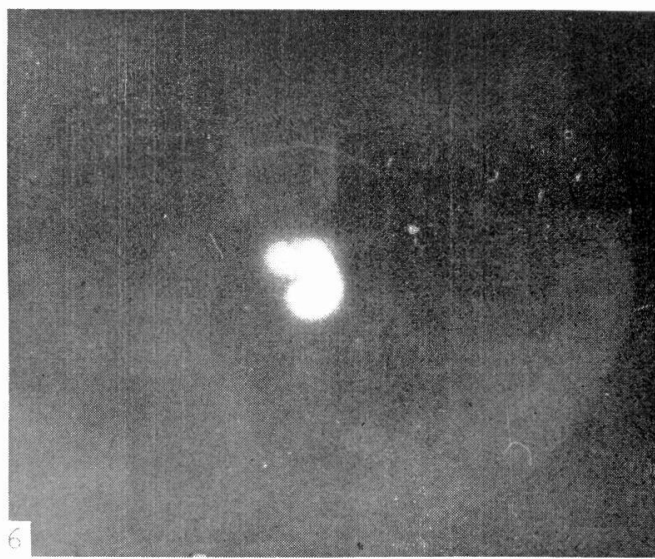

Fig. 16. The tip of this fluorescence is extremely small and short elongation. The dye gradually enlarge and become to fill the entire subretinal space.

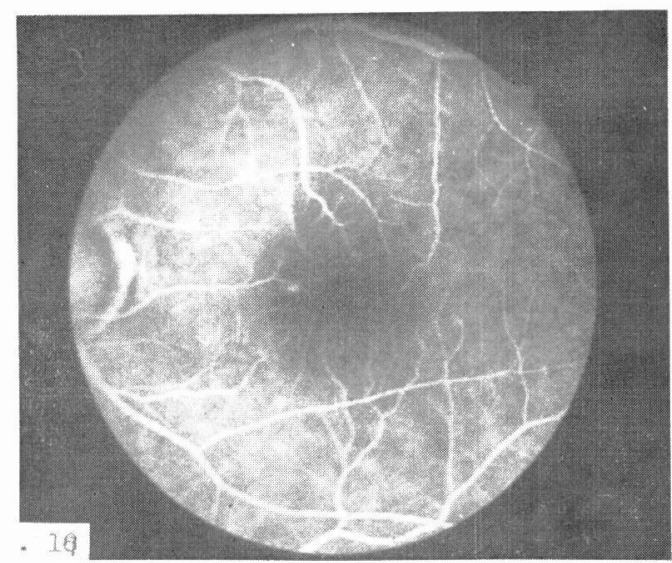

Fig. 17. Case 2. 115th day after onset, in arterial phase the focal type of fluorescence appear but this spot do not enlarge and remain in time. The spot previously jeted in the focal area is obscure.

the dye in this spot begin to jet minimally temporal inferiorly (Fig. 15). The tip of this fluorescence is extremely small and short elongation. This fluorescence gradually enlarge and thirty minutes later become to fill the entire subretinal space (Fig. 16). 76th day after onset; in the same area the abnormal fluorescein spot appear in the arterial phase and begin to jet temporal inferiorly. 
But this jeting dye only enlarge to describe a parabola and this finding consider of the transition to the next stage. Nine minutes later, the dye fill the entire detached area. 115th day after onset; in arterial phase the focal type of fluorescence appear but this spot do not enlarge and remain in time (Fig. 17). The spot previously jeted in the focal area is obscure.

Case 3. 38-year-old man

He complained central blurred vision in his right eye. The vision on the initial examination was 0.6 uncorrected. Funduscopy revealed a round circumscribed detachment of the macula. Foveal reflex was absent. There was many yellowish gray punctate deposits just temporal to superior to the foveal region (Fig. 18). Vision in his right eye, ten days later, was reduced to 0.5 , but could be corrected to 0.7 . There was still shallow serous detachment of the macula.

Fluorescein fundus findings. - 5th day after onset; the spot of abnormal fluorescence appear under the venule in the area of temporal superior to the fovea in arterial phase. At the same time, mound fluorescence of heart in shape appear in the area of nasal superior to the fovea as mottling with distinct margin. The former is focal type of detachment in pigment epithelium and the latter is mound type of it. In time, fluorescein spot of focal type slightly enlarge in shape, then jet superiorly, and enlarge to nasal superior margin of the detached retina. The spot of fluorescence gradually begin to decrease in density three or four minutes after background fluorescence has disappeared. Thirty minutes later, the dye become to fill the superior half of the detached retina. Sixty minutes later, the dye fill the entire detached ratina. The fluorescence of mound type observing nasal superior to the fovea has constant in shape and retain thirty minutes after background fluorescence has all disappeared (Fig. 19, 20). Two weeks after onset; in arterial phase, focal and mound type of fluorescenc appear in the same area. The fluorescence of focal area previously jeted enlarge

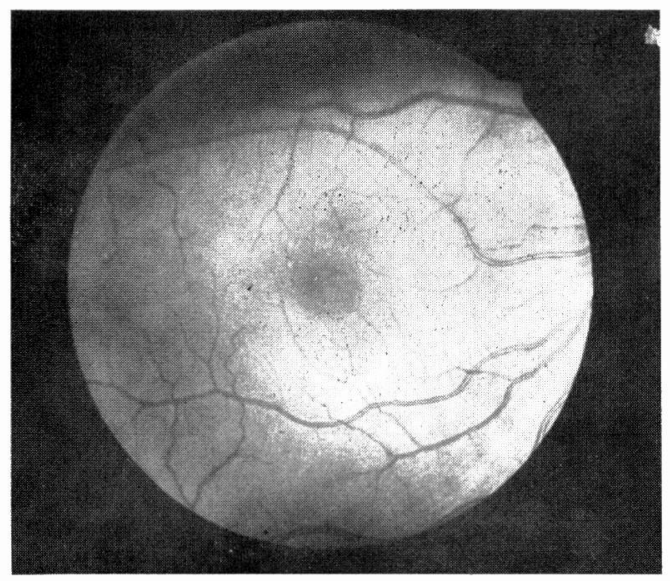

Fig. 18. Case 3. right eye. A round localized detachment of the macula. 
concentrically in time and fill the entire detached retina in thirty minutes later. The fluorescence of mound area retain as constant in shape and size. Six months after onset; fluorescence of mound area show the same findings of five days after onset. The fluorescence of focal area appear in minimal in arterial phase and disappear parallel to the background fluorescence in time (Fig. 21, 22).

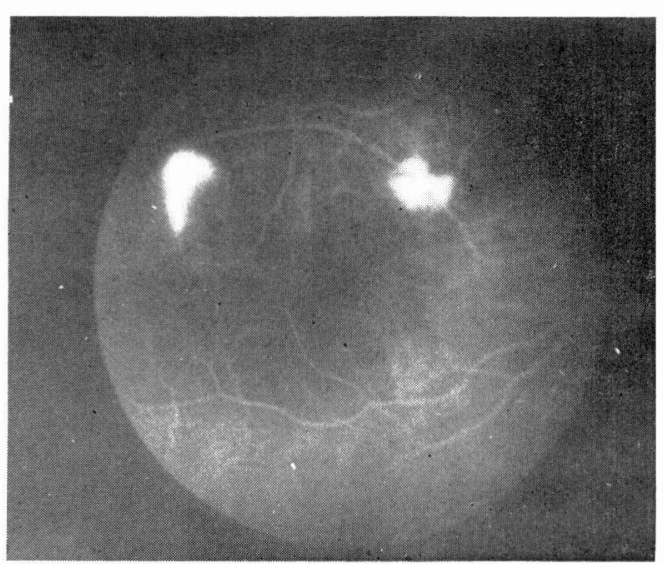

Fig. 19. Case 3. 5 th day after onset, fluorescein spot of focal type in the area of temporal superior slightly enlarge in size, then jet superiorly.

The fluorescence of mound type observing nasal superior to the fovea has constant in shape and size.

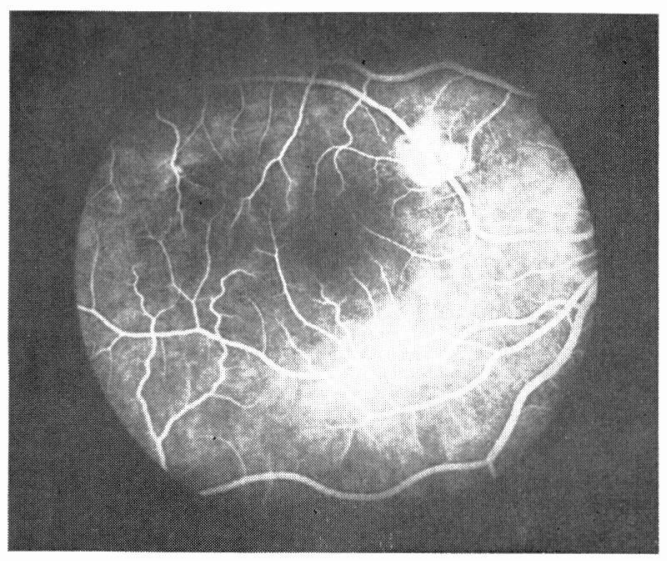

Fig. 21. Case 3. Six months after onset, fluorescence of mound area show the same findings of 5 th day after onset. The spot of focal area appear in minimal in arteriovenous phase.

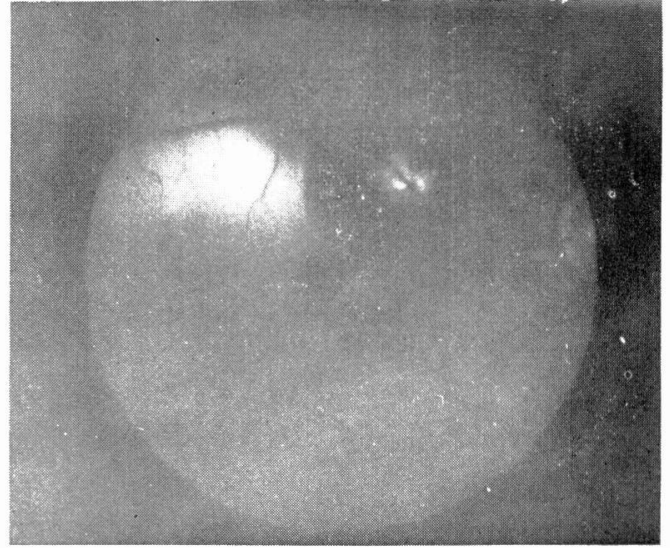

Fig. 20. Thirty minutes after injection, the fluorescence of focal area fill the entire detached retina. Otherwise, the dye of mound type observing nasal superior to the fovea has constant in shape and remain after the background fluorescence has disappeared.

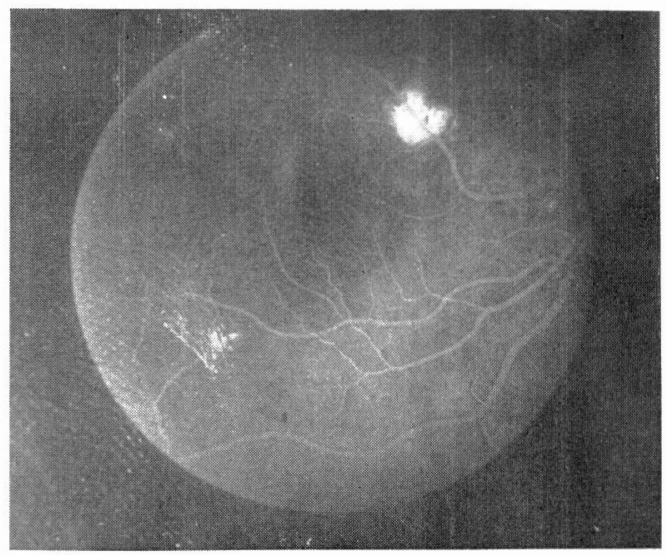

Fig. 22. 5 minutes later, the fluorescence of mound type has still constant in shape but focal type disappear parallel to the background fluorescence. 


\section{Case 4. 41-year-old man}

He noted a vague disturbance in vision, central scotoma, and metamorphopsia in his left eye. Visual acuity on the initial examination was corrected to 0.7. Funduscopy revealed loss of foveal reflex and a circumscribed round elevation of the macular region. One month later, his vision was restored to 1.0. A circumscribed retinal detachment in macular region was almost disappeared but the foveal reflex was still absent. There were several yellowish deposits in the macular area.

Fluorescein fundus findings. - 9th day after onset; the focal type of abnormal fluorescence appear in area of $1 / 2$ disc diameter temporal superior to the fovea in arterial phase. The dye jet superiorly from spot in this focal area and enlarge both side at the superior margin of the detached retina in time. Thirty minutes later, the dye further enlarge inferiorly along their margin. One month after onset; in arterial phase, abnormal fluorescence same as those previously observed appear corresponding to the pigment epithelium detachment. This area of fluorescence show mottling appearance and there are three spots that the fluorescence increase in density. One spot among these spots gradually enlarge concentrically in time and thirty minutes later the dye fill the entire subretinal space. Nine months after onset; the round fluorescein mound with mottled appearance appear in arterial phase and there are three spots in this mound. These spots of fluorescence disappears with the background fluorescence.

\section{DISCUSSION}

The course of this disease may be classified as following four stages by the method of fluorescein fundus angiography.

\section{Initial Stage}

The characteristic findings of fluorescein fundus photography in this stage are the jet type leakage of fluorescein from the choroid to the subretinal space, and then the dye always fill the entire subretinal space corresponding to the area in visible retinal detachment opthalmoscopically. When the fluorescnce is administered intravenously, the focal or mound lesion of fluorescence already appear in arterial phase, dense fluorescein spot is seen within the focal or mound lesion in arteriovenous phase, and then the dye always jets into the subretinal space upward from this spot. After thirty or sixty minutes the entire subretinal space is filled with the fluorescence but these fluorescence does not penetrate into the preretinal or retinal tissue.

Even in initial stage, the dye at first always jeting to upward become to jet sidely and then oblique inferiorly gradually in time. From these findings in this stage we prefer the word "jet type" instead of "fountain type".

These fluorescein fundus photographic findings suggest that the permeability in capillaries of the choroid accelerate, the dye leaks from the capillaries, and fill the subpigment epithelial space. The retinal pigment epithelium become to 
detach from the underlying tissue. The break may usually occur in one place of detached pigment epithelium. This break may be seen among the detached pigment epithelium, i. e. separation of terminal bar, because the first jeting time of dye is too fast after injection, the fluorescein leakage is jet type, and in some cases the spot of previously leakaged area may not be seen in healed stage.

\section{Middle Stage}

The characteristic findings of fluorescein fundus photography in this stage are the round enlarged type of fluorescein leakage in the same area as in the initial stage. The focal or mound lesion of fluorescence appear in arterial phase. The dense fluorescein spot is seen within the focal or mound lesion in arteriovenous phase and then fluorescence gradually become to enlarge roundly and in generally, these fluorescence fill the entire subretinal space. We call this stage the enlarged type.

These fluorescein fundus photographic findings suggest that fluorescein leakage from break in detached pigment epithelium in to the subretinal space has been decreased associated with diminution of capillary permeability.

\section{Late Stage}

The characteristic findings of fluorescein fundus photography in this stage may be presented that the dye do not leak to the subretinal space but only fill the residual detached pigment epithelium. When the dye is administered intravenously, the residual focal or mound lesion of fluorescence appear in arterial phase. The fluorescence in these area become more dense in time but do not change in size and form. Moreover, these fluorescence may be invariably observed after the background fluorescence has disappeared. We call this stage the residual type.

These fluorescein fundus photographic finding may be indicated that the break in the area of detachment in pigment epithelium has already disappeared and the dye may be pooled in an area of residual detached subpigment epithelium and partly among the each loosely connected pigment epithelial cell.

\section{Healed Stage}

The characteristic finding of fluorescein fundus photography in this stage is the same fluorescence pattern as the background fluorescence. We call this stage the healed stage.

This fluorescein fundus photographic finding suggest that fluorescence is transmited through the thin or depigmented area in the previously detached pigment epithelium. This finding in this stage may be consider of healed stage. Abnormal fluorescence in the full course of these cases is figured schematically (Fig. 23).

From the fluorescein fundus photographic finding $\mathrm{s}^{6}$ ) in 70 eyes of the central serous retinopathy including the cases reported here, two kinds of detachment 


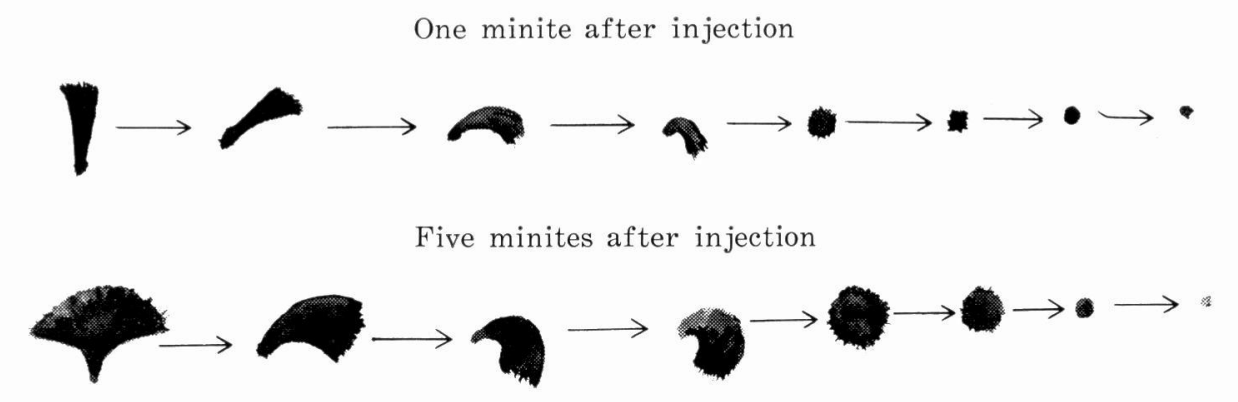

Fig. 23. Abnormal fluorescence in the full course of these cases is shown schematically.

of the retinal pigment epithelium may be seen in this disease. The first type is small in size and focal detachment of pigment epithelium (focal type). The second is relatively large or large in size and mound detachment of pigment epithelium (mound type). The course in patient with mound type may be seem to be much longer than those with focal type.

One to three fluorescein leakage from the choroid with focal or mound detachment of the pigment epithelium are the characteristic fundus features in central serous retinopathy. The spots of fluorescein leakage have never be seen in foveal region of this disease.

Therefore, fluorescein fundus photography in patient with central serous retinopathy is also very useful in deciding the stage, prognosis, and cure of this disease.

In the central serous retinopathy there may always present both of lesion with above mentioned fluorescein spot (1st lesion) and the other fluorescein spot (2nd or more lesion) at the same time. This problem will be discussed on the next paper ${ }^{6)}$.

\section{CONCLUSION}

Serial observation of the central serous retinopathy by fluorescein fundus angiography are performed in four cases. The results obtained are as follows:

1. The course of central serous retinopathy may be divided into four stages ; i. e. initial stage (Jet Type), middle stage (Enlarged Type), late stage (Residual Type) and healed stage (Constant Type), and we discussed the interpretation of fluorescein fundus angiographic findings in these each stages.

2. Two kinds of detachment type in pigment epithelium may be seen in central serous retinopathy and its size has prognostic significance.

3. Fluorescein fundus photography is very useful in deciding of the stage, prognosis, and cure of the central serous retinopathy. 


\section{ACKNOWLEDGMENT}

We wish to thank Dr. Y. Masuda of the Department of Ophthalmology for his revision and his kind advices.

\section{REFERENCES}

1) Fujisawa, Y. : Clinical studies on the retinal and choroidal lesions by fluorescein fundus photography. 1. Chorioretinitis centralis serosa. Acta Soc. Ophthal. Jap. 69, 1317-1328, 1965.

2) Fujisawa, Y. : Clinical studies on the retinal and choroidal lesion by fluorescein fundus photography. 2. Investigations following the course of central serous retinopathy. Acta Soc. Ophthal. Jap. 70, 150-160, 1966.

3) GAss, J. D. M. : Pathogenesis of disciform detachment of the neuroepithelium II. Idiopathic central serous choroidopathy. Amer. J. Ophthal. 63, 573-711, 1967.

4) JÜtтE, A. und LEMKE, L. : Chorioretinitis centralis serosa Intravitalfärbung am Augenhintergrund mit Fluoreszein-Natrium. Stuttgart, Enke, s. 71-78, 1968.

5) Wessing, A. : Ablatio centralis serosa (Retinitis centralis serosa) Fluoreszenzangiographie der Retina, Lehrbuch und Atlas. Stuttgart, Thieme, s. 104-113, 1968,

6) Yoshioka, H., Sugita, T., Naga Yoshi, K. and Endo, Y. : Fluorescein fundus photographic studies on the central serous retinopathy Part III. to be published. 